\title{
CONVERSÃO DE DAIDZINA E GENISTINA DE SOJA POR $\beta$-GLICOSIDASE DE Aspergillus oryzae
}

\author{
CLAUDIO LIMA AGUIAR * \\ YONG KUN PARK **
}

\begin{abstract}
Avaliou-se a capacidade de conversão de isoflavonas glicosiladas às suas formas agliconas pelo uso de $\beta$ glicosidase de Aspergillus oryzae ATCC 22786. A produção de daidzeína e genisteína foi acompanhada por $96 \mathrm{~h}$ de fermentação em estado sólido, usando-se farelo de soja e suspensão de esporos do fungo a $30^{\circ} \mathrm{C}$. Notou-se a conversão de glicosil-isoflavonas após $24 \mathrm{~h}$ e redução significativa na sua quantidade, depois de $48 \mathrm{~h}$ de fermentação. A atividade enzimática acompanhou a formação de produto até $72 \mathrm{~h}(0,2 \mathrm{UI} /$ $\mathrm{mL})$, apresentando leve diminuição em $96 \mathrm{~h}(0,19 \mathrm{UI} /$ $\mathrm{mL})$. Após $48 \mathrm{~h}$ obteve-se conversão total de daidzina e genistina, com formação de 551,1 e $17,2 \mu \mathrm{g} / \mathrm{g}$ de daidzeína e genisteína, respectivamente. A produção de genisteína, no entanto, mostrou-se significativamente superior em $24 \mathrm{~h}$ de fermentação $(289,3 \mu \mathrm{g} / \mathrm{g})$. Concluiuse que a $\beta$-glicosidase produzida por Aspergillus oryzae ATCC 22786 foi capaz de converter glicosilisoflavonas em agliconas por fermentação em estado sólido a $30^{\circ} \mathrm{C}$.
\end{abstract}

PALAVRAS-CHAVE: SOJA; ISOFLAVONAS; GLICOSIDASES; ASPERGILLUS.

\section{INTRODUÇÃO}

Muitas glicosidases vegetais e microbianas podem catalisar a hidrólise de glicosil-flavonóides. Tal transformação tem sido objeto de estudo de diversos pesquisadores, principalmente quanto à hidrólise de glicosilisoflavonas de soja (PARK et al., 2001a; PARK et al., 2001b; CARRÃOPANIZZI et al., 1999; ESAKI et al., 1999). Acil e glicosil hidrolases também estão envolvidas na biossíntese dos flavonóides, tais como

* Dr. em Ciência de Alimentos, Professor, Fazenda Experimental, Universidade Norte do Paraná (UNOPAR), Tamarana, PR (e-mail: claguiar@yahoo.com.br). Dr. em Ciência de Alimentos, Professor Titular, Departamento de Ciência de Alimentos, Faculdade de Engenharia de Alimentos, Universidade Estadual de Campinas, Campinas, SP. 
as $\beta$-1,4-glicosidases ( $\beta$-glicosidase, aril- $\beta$-1,4-glicosidase). Essas, muitas vezes são consideradas enzimas celulolíticas devido ao importante papel que exercem na hidrólise de produtos celulósicos (celobiose) a glicose. No entanto, há dois tipos de $\beta$-glicosidases, a aril- $\beta$-glicosidase e a $\beta$-glicosidase propriamente dita. Tais enzimas diferem quanto à especificidade de substrato, propriedades físicas e controle genético da biossíntese enzimática (GONG e TSAO, 1975).

Na soja, a atividade de glicosidase endógena tem sido percebida, e segundo MATSUURA e OBATA (1993) e MATSUURA, SASAKI e MURAO (1995) a glicosidase endógena da soja pode hidrolisar isoflavonas glicosiladas a agliconas. A hidrólise de glicosil-isoflavonas em isoflavonas agliconas e glicose, provavelmente segue o mecanismo de hidrólise de compostos glicosilados proposto por WONG (1995), e ilustrado por BLANCHARD e WITHERS (2001) (Figura 1). WONG (1995) propõe, basicamente, dois tipos de mecanismos para a hidrólise de compostos glicosilados, sendo o primeiro via um íon oxocarbônio estabilizado e o segundo por um intermediário enzima-glicosilato covalentemente ligado (WONG, 1995).

\section{FIGURA 1- MECANISMO GERAL DE CATÁLISE POR GLICOSIDASES}

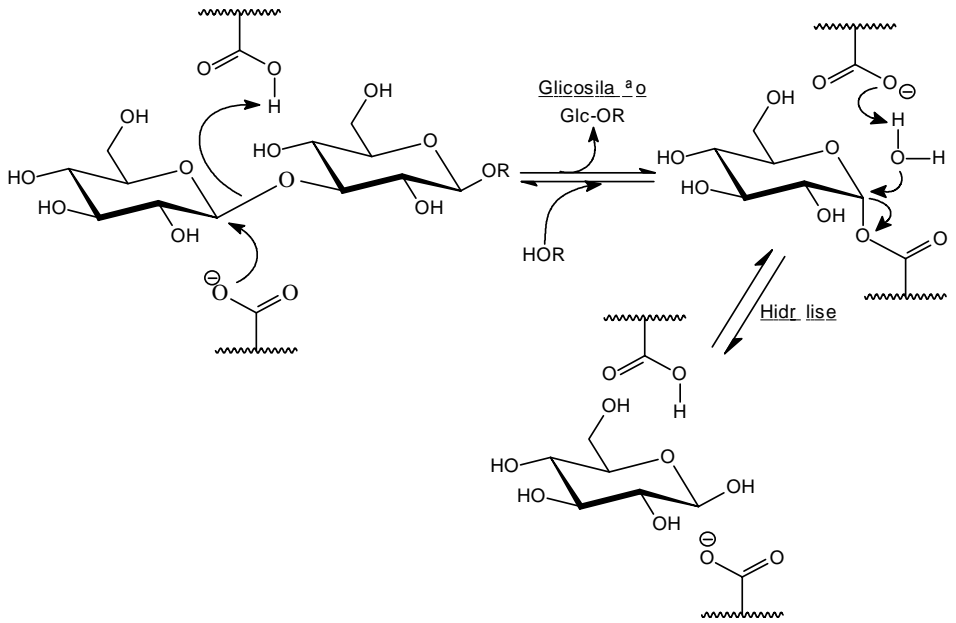

Fonte: BLANCHARD e WITHERS (2001). 
Nos vegetais, a atividade de $\beta$-glicosidase está envolvida em diversos processos. Entre esses, pode-se citar a atividade de fito-hormônios, mecanismos de defesa contra microrganismos, insetos ou parasitas, desenvolvimento e pigmentação floral, e atividade na lignificação e decomposição da parede celular (HSIEH e GRAHAM, 2001).

A transformação de isoflavonas por processos enzimáticos bem estabelecidos torna-se ainda mais interessante para países produtores de soja, como o Brasil. A soja contém grandes quantidades de isoflavonas glicosiladas passíveis de hidrólise enzimática, produzindo formas agliconas que mostraram-se mais ativas contra radicais livres, tumores da mama e próstata, entre outras atividades biológicas (PARK et al., 2001c; SETCHELL e CASSIDY, 1999).

Em grãos de soja são encontradas isoflavonas, predominantemente nas formas glicosilada, malonilglicosilada e acetilglicosilada. Durante processos térmicos e fermentativos, esses compostos são transformados às suas formas agliconas e acumulados no produto final (KLUS e BARZ, 1998). A Figura 2 mostra a transformação enzimática de glicosil-isoflavona, amplamente encontrada em grãos de soja, à sua correspondente forma aglicona (PARK et al., 2001b).

\section{FIGURA 2 - TRANSFORMAÇÃO ENZIMÁTICA DE GLICOSIL- ISOFLAVONAS A AGLICONAS}

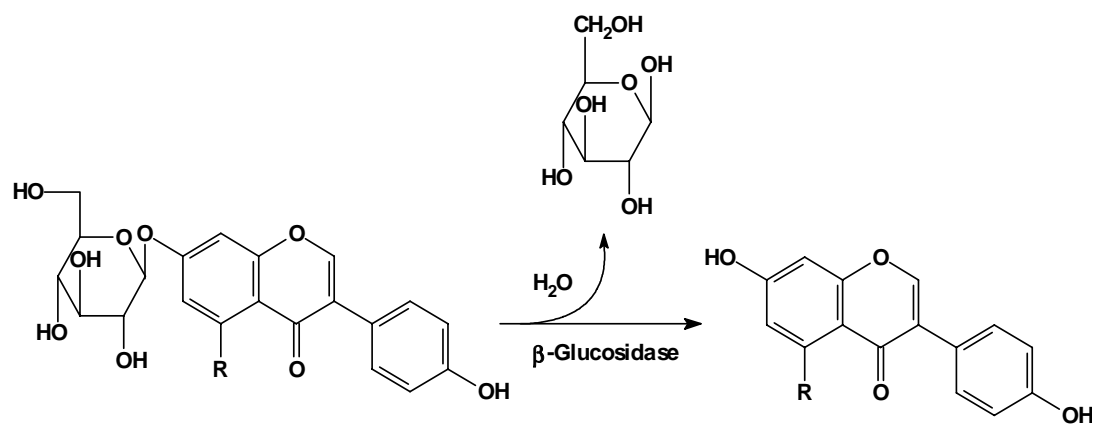

Fonte: PARK et al. (2001b). 
A associação da atividade de $\beta$-glicosidase com a hidrólise de glicosilisoflavonas, apresentada por PARK et al. (2001a, b), foi confirmada por outros pesquisadores como IBE et al. (2001). Esses, perceberam que uma bactéria isolada de palhas de arroz e identificada por IFO 9916 foi capaz de converter grande quantidade de glicosil-isoflavonas às suas correspondentes formas agliconas pela ação de $\beta$-glicosidase. Da mesma forma, MATSUDA et al. (1994) reportaram que a $\beta$-glicosidase de Lactobacillus casei subsp. rhamnosus IFO 3425 mostrou grande capacidade hidrolítica de glicosil-isoflavonas em xarope cozido de soja.

Tem-se notado que produtos não-fermentados de soja contém menor teor de isoflavonas agliconas do que os fermentados. Nos produtos fermentados, como "misso" e "tempeh", a conversão de glicosilisoflavonas está associada à presença de $\beta$-glicosidase. No entanto, em outro produto fermentado denominado "natto", os teores de isoflavonas agliconas são similares aos de glicosilados. Pode-se então presumir que Bacillus natto (bactéria responsável pela fermentação) não produziria $\beta$-glicosidase. Outro fato observado foi o alto teor de glicosil-isoflavonas em leite fermentado de soja em relação às agliconas. A causa seria fermentação ineficiente do leite de soja pelas bactérias ácido-lácticas (Lactobacillus bulgaricuse Streptococcus thermophilus), ou ainda, a não-produção de $\beta$-glicosidase por essas bactérias (KIYOSAWA et al., 1995). De acordo com CARRÃO-PANIZZI e BORDINGNON (2000), $\beta$-glicosidase endógena de soja também é capaz de hidrolisar glicosil-isoflavonas à agliconas.

A conversão de glicosil-isoflavonas à agliconas tem sido feita principalmente pela utilização de $\beta$-glicosidase, embora diversos trabalhos demonstrem a possibilidade de ocorrer outras enzimas que agem diferentemente. MATSUURA e OBATA (1993), por exemplo, purificaram três isoenzimas de $\beta$-glicosidase de soja com capacidade de hidrolisar glicosil-isoflavonas presentes em leite de soja. MATSUURA, SASAKI e MURAO (1995) observaram que uma $\beta$-glicosidase foi capaz de hidrolisar daidzina e genistina, produzindo daidzeína e genisteína. Além das diferentes isoformas de $\beta$-glicosidase, outra enzima denominada lactase florizina hidrolase (EC 3.2.1.62) encontrada no intestino de mamíferos foi capaz de hidrolisar alguns tipos de isoflavonas e flavonóis glicosilados à suas formas agliconas (DAY et al., 2000). A hidrólise de flavonóides como rutina, forma 
glicosilada da quercetina, foi observada por NARIKAWA, SHINOYAMA e FUJII (2000). Anteriormente, NARIKAWA (1998) reportaram que algumas glicosidases foram produzidas em cultura líquida contendo rutina como indutor. Penicillium rugulosum IFO 7242 produziu $\beta$-rutinosidase capaz de hidrolisar rutina em rutinose e quercetina. Deve-se ressaltar que essa glicosidase não apresentou atividade sobre 4-nitrofenil $\beta$-glicosídeo ou celobiose, os quais constituem tradicionais substratos para glicosidases.

Isoflavonas agliconas apresentam maior capacidade no controle de células cancerosas, inibindo o crescimento de células tumorais da próstata humana quando comparada às suas formas glicosiladas (MATSUDA et al., 1994). Além disso, foi reportado que a atividade antioxidante de genisteína ou daidzeína mostrou-se superior à de suas formas glicosiladas (ONOZAWA et al., 1998). A conversão de $\beta$-glicosil à isoflavonas agliconas resulta em quantidades maiores de compostos benéficos à saúde humana.

Neste trabalho foi estudada a produção de $\beta$-glicosidase por Aspergillus oryzae ATCC 22786, Aspergillus niger e Aspergillus awamori, bem como a capacidade da b-glicosidase produzida converter formas glicosiladas de isoflavonas presentes em farelo de soja às suas correspondentes formas agliconas.

\section{MATERIAL E MÉTODOS}

\subsection{MATERIAL}

A água utilizada nas análises cromatográficas foi deionizada em colunas de troca-iônica (Millipore do Brasil) e filtrada através de membranas de $0,22 \mu \mathrm{m}$ (Millipore do Brasil). Empregaram-se ácido acético e metanol (fase móvel) grau cromatográfico e solventes orgânicos (extração das isoflavonas) de grau analítico. Daidzeína (sintética; 98\%) e genisteína (sintética; 98\%) foram obtidas da Sigma Chemical Co. (St. Louis, USA), daidzina (de soja; 99\%) e genistina (de soja; 99\%) foram doadas pela Funakoshi Co. (Japão). Os reagentes para determinação da atividade enzimática de $\beta$-glicosidase foram adquiridos da Sigma Chemical Co. (St. Louis, USA). O farelo de soja foi obtido a partir de grãos de soja, cultivar BR-36, doados pela Gebana Co. (Capanema, PR) e submetidos à moagem em moído de facas. 


\subsection{PRODUÇÃO DA ENZIMA}

Diferentes cepas dos fungos filamentosos, Aspergillus oryzae, Aspergillus niger e Aspergillus awamori foram avaliados quanto à produção de $\beta$-glicosidase. Para a fermentação em estado sólido foram utilizados frascos de Erlenmeyer de $500 \mathrm{~mL}$, contendo $20 \mathrm{~g}$ de farelo de soja. Após esterilização, os frascos com o farelo foram inoculados com um mililitro de suspensão de esporos $\left(10^{7}\right.$ esporos $\left./ \mathrm{mL}\right)$ do microrganismo e incubados em estufa a $30^{\circ} \mathrm{C}$ durante $24,48,72$ e $96 \mathrm{~h}$ e controle (tempo zero). Decorridos os períodos de incubação foram adicionados $100 \mathrm{~mL}$ de água destilada aos frascos, sendo o meio de cultura homogenizado com auxílio de bastão de vidro. Filtrouse a suspensão obtida em lã de vidro para remoção do micélio fúngico.

Determinou-se a atividade de $\beta$-glicosidase conforme descrito por MATSUURA, SASAKI e MURAO (1995). A mistura de $2 \mathrm{~mL}$ de solução $1 \mathrm{mM}$ de $p$-nitrofenil- $\beta$-D-glicopiranosídeo (pNPG) em tampão fosfatocitrato $0,1 \mathrm{M} \mathrm{e} \mathrm{pH} 5,0$ foi pré-incubada a $40^{\circ} \mathrm{C}$ por 5 minutos. Após a adição de $0,5 \mathrm{~mL}$ de extrato enzimático, a mistura foi incubada a $40^{\circ} \mathrm{C}$ por 20 minutos. Interrompeu-se a reação pela adição de 2,5 $\mathrm{mL}$ de carbonato de sódio $0,5 \mathrm{M}$ e mediu-se a absorbância do sistema reacional a $420 \mathrm{~nm}$ (Espectrofotômetro Beckman Coulter DU-70, Estados Unidos). A quantidade de $p$-nitrofenol liberada que forma um íon de coloração amarelada foi determinada mediante curva-padrão, preparada da mesma maneira, substituindo-se os extratos enzimáticos por soluções contendo concentrações crescentes (5-300 mmol) de $p$ nitrofenol, adicionadas à solução-tampão fosfato-citrato $0,1 \mathrm{M}(\mathrm{pH} 5,0)$ e carbonato de sódio $0,5 \mathrm{M}$.

\subsection{DETERMINAÇÃO DAS ISOFLAVONAS}

Os farelos de soja secos foram moídos até obtenção da farinha (200 mesh), que foi desengordurada com hexano $(1: 10, \mathrm{~m} / \mathrm{v})$ por 30 minutos a $25^{\circ} \mathrm{C}$ sob agitação rotativa. Misturou-se a farinha desengordurada de soja com $10 \mathrm{~mL}$ de solução $80 \%$ de metanol por uma hora a $25^{\circ} \mathrm{C}$ para extração das isoflavonas totais. Utilizou-se o sobrenadante para determinação do teor de isoflavonas em cromatografia a líquido de alta eficiência em fase reversa (CLAE-FR) de acordo com o método proposto por KOO et al. (2000), modificado por PARK et al. (2002). Com fluxo 
de $0,5 \mathrm{~mL} / \mathrm{min}$ foram injetadas alíquotas de vinte microlitros em cromatógrafo LC-10Avp, equipado com coluna YMC Pack ODS-A (RP18; comprimento de 4,6 x $250 \mathrm{~mm}$; partículas de $5 \mu \mathrm{m}$ e $120 \AA$ A ) e detector de arranjo de diodos (SPD-M10A, Shimadzu Co., Japão). A utilizando gradiente linear de água-ácido acético (solvente $A ; 19: 1, v / v$ ) e metanol (solvente $B$ ), o sobrenadante foi seletivamente eluído. $O$ gradiente foi iniciado com $20 \%$ da solução B, passando para $40 \%$ em $15 \mathrm{~min}$, subindo a $50 \%$ entre 15 e $55 \mathrm{~min}$, atingindo $80 \%$ em $95 \mathrm{~min}$ e decrescendo para $20 \%$ entre 95 e $105 \mathrm{~min}$. A coluna foi reequilibrada com $20 \%$ da solução B por 15 min entre as análises. As isoflavonas eluídas foram detectadas por absorbância a $254 \mathrm{~nm}$. Análise quantitativa de daidzina, genistina e suas formas agliconas foram obtidas por comparação com padrões autênticos. As concentrações foram expressas em $\mu \mathrm{g}$ de isoflavonas por $\mathrm{g}$ de farelo de soja, em base seca.

\section{RESULTADOS E DISCUSSÃO}

As atividades enzimáticas da $\beta$-glicosidase dos diferentes fungos utilizados são mostradas na Tabela 1.

\section{TABELA 1 - ATIVIDADES ENZIMÁTICAS DE b-GLICOSIDASE}

\begin{tabular}{lccc}
\hline Amostras & Fonte & Cole ${ }^{{ }^{\mathbf{a}} \mathbf{0}}$ & ${ }^{\mathbf{a}}{\mathrm{Ul} \mathbf{~}^{-1}}^{-1}$ \\
\hline Aspergillus oryzae ATCC 22786 & Arroz Koji & ${ }^{\circ}$ ATCC & 0,18 \\
Aspergillus oryzae II & Solo & ${ }^{\mathrm{c}}$ DCA-UNICAMP & 0,35 \\
Aspergillus niger I & Solo & DCA-UNICAMP & 0,46 \\
Aspergillus niger II & Alimentos amil/Eeos & DCA-UNICAMP & 0,14 \\
Aspergillus awamori ATCC 22342 & Fibras & ATCC & 0,15 \\
\hline
\end{tabular}

a Atividade enzimática da $\beta$-glicosidase medida contra PNPG, depois do crescimento do fungo em meio semi-sólido de farinha de soja e água destilada $(1: 1, \mathrm{p} / \mathrm{v})$ a $30^{\circ} \mathrm{C}$ e 96 horas.

${ }^{b}$ American Type Culture Collection.

c Fungos constantes da coleção do Laboratório de Bioquímica de Alimentos da Universidade Estadual de Campinas (Campinas, Brasil).

A atividade para a $\beta$-glicosidase de Aspergillus oryzae ATCC 22786 mostrou-se inferior à de outros fungos utilizados neste estudo. No 
entanto, sua aplicabilidade seria maior devido ao fato de ter sido isolado de produtos da fabricação de "Amazake", principalmente de arrozkoji. Além disso, trata-se de organismo seguro para a saúde humana (Generally Recognized As Safe - GRAS), não-produtor de aflatoxina (nível de segurança BSL 1), ou seja, não causa enfermidades em humanos adultos. Esse fungo, A. oryzae ATCC 22786, foi isolado inicialmente no Japão, e tem outras classificações de acordo com as normas da Coleção em que está depositado (RIB 430, CBS 816.72 e IFO 30104).

Em diferentes alimentos à base de soja, tal como leite de soja em pó, as isoflavonas apresentam-se na forma glicosilada, posto que a farinha de soja já sofreu algum tipo de tratamento térmico durante 0 processamento industrial para a remoção do óleo. Vários estudos têm demonstrado que as isoflavonas agliconas apresentam maior atividade antioxidante, ressaltando a importância de se estudar os métodos de conversão das formas glicosiladas em agliconas. A conversão de glicosil isoflavonas em agliconas, seja por meio de fermentação em estado sólido ou aplicação de enzima (endógena ou exógena), estaria relacionada à presença de $\beta$-glicosidase. De fato, os esporos de $A$. oryzae ATCC 22786 inoculados sobre farelo de soja germinaram e a atividade enzimática de $\beta$-glicosidase foi observada em 24 horas de fermentação a $30^{\circ} \mathrm{C}$. Durante a fermentação do farelo de soja por 48 horas (Tabela 2), a maioria dos $724 \mu \mathrm{g}$ de glicosil isoflavonas foi convertida em suas correspondentes formas agliconas $(564 \mu \mathrm{g})$, considerando um grama de farelo de soja, em base seca. Tornou-se evidente que a conversão ocorreu principalmente pelo aumento da atividade enzimática após de 24 horas de fermentação, e a produção da enzima apresentou-se associada à formação de daidzeína e genisteína (Tabela 2 e Figura 3).

Durante a fermentação com esporos do fungo, a quantidade de isoflavonas glicosiladas diminuiu significativamente após $48 \mathrm{~h}$ com pequena perda das isoflavonas totais (Tabela 2). A transformação de daidzina e genistina foi completa com formação de $930,9 \mu \mathrm{g} / \mathrm{g}$ de daidzeína e de 17,2 $\mu \mathrm{g} / \mathrm{g}$ de genisteína em 96 h. A produção de genisteína, no entanto, mostrou-se significativamente superior em $24 \mathrm{~h}$ de fermentação $(398,3 \mu \mathrm{g} / \mathrm{g})$. Verificou-se redução no conteúdo de genisteína ao longo da fermentação, possivelmente, relacionada com reações paralelas. Tratando-se de fermentação semi-sólida podem 
ocorrer outras vias catabólitas desse isoflavonóide. Muitos microrganismos apresentam a capacidade de metabolizar flavonóides e suas estruturas modificadas têm sido encontradas. Flavonóides podem ser modificados por diferentes reações, ocorrendo a hidroxilação, metilação, glicosilação e acetilação com quase todas as classes de flavonóides (HELLER e FORKMANN, 1988).

\section{TABELA 2 - VARIAÇÃO DO CONTEÚDO DE DAIDZINA E GENISTINA DURANTE FERMENTAÇÃO SEMI-SÓLIDA DE FARINHA DE SOJA COM ESPOROS DE Aspergillus oryzae ATCC 22786}

\begin{tabular}{lccccc}
\hline Isoflavonas & \multicolumn{5}{c}{$\mu \mathrm{g}$ isoflavonas/g soja } \\
\cline { 2 - 6 } & Controle $^{\circ}$ & $24 \mathrm{~h}$ & $48 \mathrm{~h}$ & $72 \mathrm{~h}$ & $96 \mathrm{~h}$ \\
\hline Daidzina & 244,9 & 26,0 & 0,0 & 0,0 & 0,0 \\
Genistina & 309,9 & 64,6 & 0,0 & 0,0 & 0,0 \\
Daidze na & 265,0 & 558,7 & 816,1 & 906,1 & 1195,9 \\
Geniste na & 418,0 & 707,3 & 386,0 & 392,2 & 435,2 \\
\hline
\end{tabular}

Temperatura de fermentação mantida em $30 \pm 1^{\circ} \mathrm{C}$.

${ }^{a}$ Média dos valores de três repetições.

${ }^{b}$ Incubação em solução tampão citrato-fosfato $50 \mathrm{mM}$ e pH 5,4, sem a presença de esporos fúngicos.

FIGURA 3 - PRODUÇÃO DE $\beta$-GLICOSIDASE DE Aspergillus oryzae ATCC 22786 POR FERMENTAÇÃO EM ESTADO SÓLIDO SOBRE FARELO DE SOJA

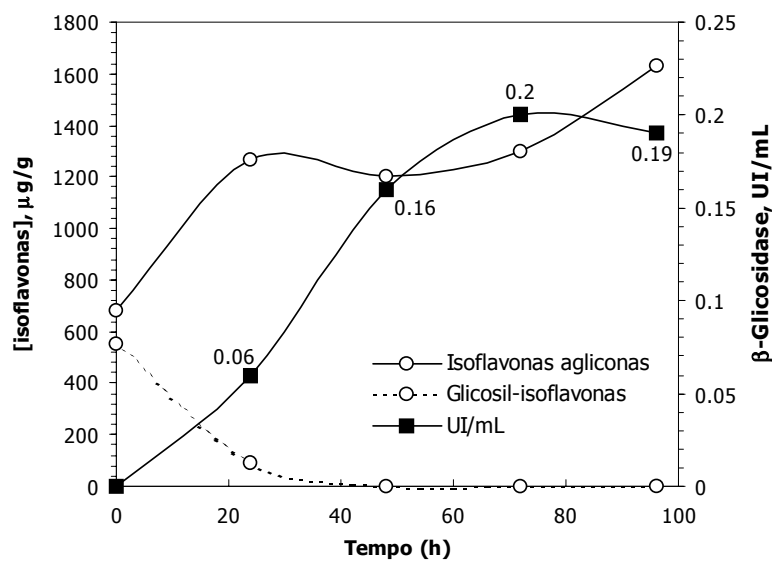


Como já estudado para outros fungos e bactérias (ESAKI et al., 1999; ESAKI, ONOZAKI e MORIMITSU, 1998; MATSUURA, SASAKI e MURAO, 1995; IKEDA, OHTA e WATANABE, 1995 e MATSUURA e OBATA, 1993), este trabalho propôs associar a atividade de $\beta$-glicosidase com a transformação de isoflavonas glicosiladas para suas formas agliconas durante a fermentação em estado sólido usando farelo de soja e A. oryzae ATCC 22786. PARK et al. (2001a; 2001c) também obtiveram isoflavonas agliconas após utilização de $\beta$-glicosidase em extrato concentrado de isoflavonas de soja. Em estudos anteriores foi demonstrado que as isoflavonas agliconas apresentam maiores atividades biológicas, tais como antioxidante e anti-inflamatória.

\section{CONCLUSÃO}

Certamente a soja constitui importante fonte de isoflavonas glicosiladas passíveis de conversão às suas formas agliconas. Os resultados obtidos permitiram concluir que $\beta$-glicosidase de Aspergillus oryzae ATCC 22786 foi capaz de hidrolisar glicosil-isoflavonas presentes em farelo de soja à agliconas, após 24 horas de fermentação em estado sólido a $30^{\circ} \mathrm{C}$. A formação dos produtos (daidzeína e genisteína) foi associada à produção de $\beta$-glicosidase até $72 \mathrm{~h}$, com leve diminuição da atividade com $96 \mathrm{~h}$ de fermentação.

\section{Abstract}

\section{CONVERSION OF SOY DAIDZEIN AND GENISTEIN BY $\beta$-GLUCOSIDASE OF Aspergillus oryzae}

The conversion capacity of glucoside isoflavones to aglycones by Aspergillus oryzae ATCC $2786 \beta$-glucosidase was evaluated. The production of daidzein and genistein was observed for $96 \mathrm{~h}$ in solid state fermentation, by utilizing soy flour and a suspension of fungal spores at $30^{\circ} \mathrm{C}$. The conversion of glucoside isoflavones was noted after $24 \mathrm{~h}$ and a significant reduction in its quantity after $48 \mathrm{~h}$ of fermentation. The enzymatic activity followed the product formation until $72 \mathrm{~h}$ $(0.2 \mathrm{UI} / \mathrm{mL})$, showing a slight reduction in $96 \mathrm{~h}(0.19 \mathrm{UI} / \mathrm{mL})$. After $48 \mathrm{~h}$ total conversion of daidzein and genistein was obtained, with formation of 551.1 and $17.2 \mu \mathrm{g} / \mathrm{g}$ of daidzein and genistein, respectively. The production of genistein, however, was significantly higher in $24 \mathrm{~h}$ of fermentation $(289.3 \mu \mathrm{g} / \mathrm{g})$. It was concluded that the produced $\beta$-glucosidase by Aspergillus oryzae was capable to convert glucoside isoflavones in to aglycones by solid state fermentation at $30^{\circ} \mathrm{C}$.

KEY-WORDS: SOY; ISOFLAVONES; GLUCOSIDASES; Aspergillus. 


\section{REFERÊNCIAS}

1 BLANCHARD, J.E.; WITHERS, S.G. Rapid screening of the aglycone specificity of glycosidases: applications to enzymatic synthesis of oligosaccharides. Chemistry and Biology, v. 8, n. 7, p. 627-633, 2001.

2 CARRÃO-PANIZZI, M.C.; BORDINGNON, J.R. Activity of beta-glucosidase and levels of isoflavone glucosides in soybean cultivars affected by the environment. Pesquisa Agropecuária Brasileira, v. 35, n. 5, p. 873-878, 2000.

3 CARRÃO-PANIZZI, M.C.; BELÉIA, A.P.; KITAMURA, K., OLIVEIRA, M.C.N. Effects of genetics and environment on isoflavone content of soybean from different regions of Brazil. Pesquisa Agropecuária Brasileira, v. 34, n. 10, p. 1787-1795, 1999.

DAY, A. J.; CANADA, F. J.; DIAZ, J. C.; KROON, P. A.; MCLAUCHLAN, R.; FAULDS, C. B.; PLUMB, G. W.; MORGAN, M. R. A.; WILLIAMSON, G. Dietary flavonoid and isoflavone glycosides are hydrolysed by the lactase site of lactase phlorizin hydrolase. FEBS Letters, v. 468, n. 2-3, p. 166, 2000.

ESAKI, H.; WATANABE, R.; ONOZAKI, H.; KAWAKISHI, S.; OSAWA, T. Formation mechanism for potent antioxadative O-dihydroxyisoflavones in soybean fermented with Aspergillus saitoi. Bioscience, Biotechnology, Biochemistry, v. 63, n. 5, p. 851-858, 1999.

ESAKI, H.; ONOZAKI, H.; MORIMITSU, Y. Potent antioxidative isoflavones isolate from soybeans fermented with Aspergillus saitoi. Bioscience, Biotechnology, Biochemistry, v. 62, n. 4, p. 740-746, 1998.

GONG, C.S.; TSAO, G.T. Cellulase and biosynthesis regulation. Annual Reports on Fermentation Processes, v. 3 (supl.), p. 111-139, 1975.

HELLER, W.; FORKMANN, G. The flavonoids: advances in research since 1980. London: Chapman and Hall, 1988. p. 399.

HSIEH, M.C.; GRAHAM, T.L. Partial purification and characterization of a soybean $\beta$-glucosidase with high specific activity towards isoflavone conjugates. Phytochemistry, v. 58, n. 7, p. 995-1005, 2001.

IBE, S.; KUMADA, K.; YOSHIBA, M.; ONGA, T. Production of natto which contains a high level of isoflavone aglycones. Journal of the Japanese Society for Food Science and Technology, v. 48, n. 1, p. 27-34, 2001.

IKEDA, R.; OHTA, N.; WATANABE, T. Changes of isoflavones at various stages of fermentation in defatted soybeans. Journal of the Japanese Society for Food Science and Technology, v. 42, n. 5, p. 322-327, 1995.

KIYOSAWA, I.; MATSUYAMA, J.; ARAI, C.; SETOGUCHI, T. Suppressive effects of the methanolic extracts from soybean products on SOS response 
of Salmonella typhimurium induced by mutagens and their contents of isoflavones. Journal of the Japanese Society for Food Science and Technology, v. 42, n. 10, p. 835-842, 1995.

KLUS, K.; BARZ, W. Formation of polyhydroxylated isoflavones from the isoflavones genistein and biochanin A by bacteria isolated from tempeh. Phytochemistry, v. 47, n. 6, p. 1045-1048, 1998.

14 KOO, H.; ROSALEN, P.L.; CURY, J.A.; AMBROSANO, G.M.B.; MURATA, R.M.; YATSUDA, R.; IKEGAKI, M.; ALENCAR, S.M.; PARK, Y.K. Effect of a new variety of Apis mellifera propolis on mutans streptococci. Current Microbiology, v. 41, n. 3, p. 192-196, 2000.

MATSUDA, S.; NORIMOTO, F.; MATSUMOTO, Y.; OHBA, R.; TERAMOTO, Y.; OHTA, N.; UEDA, S. Solubilization of novel isoflavone glycoside hydrolyzing $\beta$-glucosidase from Lactobacillus casei subsp. rhamnosus. Journal of Fermentation and Bioengineering, v. 77, n. 4, p. 439-441, 1994.

MATSUURA, M.; SASAKI, J.; MURAO, S. Studies on $\beta$-glucosidase from soybeans that hydrolyze daidzin and genistin: isolation and characterization of an isozyme. Bioscience, Biotechnology, Biochemistry, v. 59, n. 5, p. 1623-1627, 1995.

17 MATSUURA, M.; OBATA, A. $\beta$-Glucosidases from soybeans hydrolyze daidzin and genistin. Journal of Food Science, v. 58, n. 1, p. 144-147, 1993.

18 NARIKAWA, T.; SHINOYAMA, H.; FUJII, T. A $\beta$-rutinosidase from Penicillium rugulosum IFO 7242 that is a peculiar flavonoid glycosidase. Bioscience, Biotechnology, and Biochemistry, v. 64, n. 6, p. 1317-1319, 2000.

NARIKAWA, T.; KARAKI, Y.; SHINOYAMA, H.; FUJII, T. Rutin degradation by culture filtrates from penicillia. Journal of the Japanese Society for Food Science and Technology, v. 72, n. 2, p. 473-479, 1998.

20 ONOZAWA, M.; FUKUDA, K.; OHTANI, M.; AKAZA, H.; SUGIMURA, T.; WAKABAYASHI, K. Effects of soybean isoflavones on cell growth and apoptosis of the human prostatic cancer cell line LNCaP. Japanese Journal of Clinical Oncology, v. 28, n. 6, p. 360-363, 1998.

21 PARK, Y. K.; AGUIAR, C. L.; ALENCAR, S. M.; MASCARENHAS, H. A. A.; SCAMPARINI, A. R. P. Conversão de malonil-beta-glicosil isoflavonas em isoflavonas glicosiladas presentes em alguns cultivares de soja brasileira. Ciência e Tecnologia de Alimentos, v. 22, n. 2, p. 130-135, 2002.

PARK, Y.K.; ALENCAR, S.M.; NERY, I.A.; AGUIAR, C.L.; PACHECO, T.A.R.C. Enrichment of isoflavone aglycones in extracted soybean isoflavones by heat and fungal $\beta$-glucosidase. Food and Science Industry, v. 34, n. 4, p. 14-19, 2001a. 
23 PARK, Y.K.; AGUIAR, C.L.; ALENCAR, S.M.; SCAMPARINI, A.R.P. Biotransformação de $\beta$-glicosil isoflavonas de soja em isoflavonas agliconas por $\beta$-glicosidase fúngica. Documentos da Embrapa, v. 169, n. 1, p. 3336, 2001b.

24 PARK, Y.K.; AGUIAR, C.L.; ALENCAR, S.M.; MASCARENHAS, H.A.A.; SCAMPARINI, A.R.P. Survey of isoflavone contents in Brazilian soybean. Ciencia y Tecnología Alimentaria, v. 3, n. 3, p. 156-160, 2001c.

25 SETCHELL, K. D. R.; CASSIDY, A. Dietary isoflavones: biological effects and relevance to human health. Journal of Nutrition, v. 129 (supl.), p. 758-767, 1999.

26 WONG, D.W.S. Food enzymes: structure and mechanism. London: Chapman and Hall, 1995. p. 85-123. 